\title{
The Measurement of Urbanization Level Based on Entity Space: A Case Study of JingJinJi Region, China
}

\author{
Fenghua Wen ${ }^{1,2, *}$ and Lirong Ren ${ }^{2}$ \\ ${ }^{1}$ School of National Fiscal Development, Beijing, China \\ ${ }^{2}$ School of Government, Central University of Finance and Economics, Beijing, China \\ Corresponding author: wen_fh@163.com
}

\begin{abstract}
On the basis of analyzing the pros and cons of different urbanization level measurement methods, this paper puts forward urbanization level measurement method based on entity space and then uses entity space data to describe the urbanization rate of various districts and counties of JingJinJi Region in 2005 and 2009. According to the analysis of time and space differences on such basis, JingJinJi may be divided from high to low order according to the urbanization rate of the entity space: central urban area, urban development area and suburb, which respectively bear different functions. According to the comparison of entity space urbanization rate and population urbanization rate in the aspects of sequence, primacy ratio, zipf index, etc., we find the non-intensive land use status quo of urban development area of JingJinJi and the feature that the distribution of entity space urbanization scale is much uniform than distribution of population urbanization scale. Accordingly, the paper puts forward the following suggestions : (1) The space construction should depend upon function division; (2) The urban development area should pay attention to the construction of the supporting facilities and meanwhile enhance land use rate; (3) The urban construction should be enhanced in small and middle regions and counties surrounding Beijing and Tianjin.
\end{abstract}

\section{Introduction}

Karl Heinrich Marx firstly mentioned "rural urbanization" [1] when discussing urban and rural division and urban development in the Critique of Political Economy in 1858. In the following 1867, the Spanish Engineer Serda definitely proposed the conception of city urbanization in Basic Principle of City Urbanization and the conception became popular as world urbanization accelerated. Chinese scholars extended the conception as "urbanization" in consideration of Chinese actual conditions [2]. In regards to domestic definition of urbanization, the principles and methods of different subjects are different. Population urbanization refers to the population gathering course towards city and is the core of the urbanization [3]; land urbanization is the carrier of the urbanization and is the enhancement of urban space extension and land use efficiency enhancement in essence [4]; economic urbanization is the power of the urbanization and is the transition of economic structure in essence; social urbanization is the inevitable result of the urbanization and is population, land and economy urbanization and the change course of people's production form, life form, behavior habits and values [5]; ecological urbanization is the development form of the urbanization and is the external extension of new-type urbanization under sustainable development view ${ }^{[6]}$. To sum up, urbanization [7] is comprehensive urbanization of population scale, space extension, economic form, industrial structure, infrastructure construction and resource and environment support, etc.

The urbanization level measurement is one of important contents of urbanization research. Different subjects have different definitions about urbanization level. Accordingly, the measurement methods of the urbanization level show diversified. The urbanization level measured based on traditional population, land, economy, society and ecology has respective advantages and disadvantages. What's popularly used in the educational circles is the ratio of the urban population to the total population, namely population urbanization. The indicator is simple, clear, and easy for statistics and disposal and is of great practicability and is the mainstream method [8] to measure the urbanization level at present. The urban population here is also divided into registered population and permanent resident population. Different statistics methods may lead to different results. In addition, the change of the administrative division and population mobility also leads to the result difference [9]. Therefore, the educational circles also propose to use land measurement method and indicators such as economy, society, and ecology to measure the urbanization level. Land urbanization level refers to the ratio of urbanization area to the total area, namely urbanization level = (area of finished region/total area) $\mathrm{x} 100 \%$. The measurement method is simple and high pragmatic and can reflect the urbanization level in the angle of land use. The measurement result comparatively conforms to the facts. However, it may be influenced by population 
density, construction speed and quality of the urban area as well as the geological technology, especially monitoring and statistics of remote sensing technology. The data acquisition is difficult. In general, compound measurement method is adapted to measure economic, social and ecological urbanization level. For instance, the indicators of economic urbanization include ratio of the tertiary industry to GDP, total annual fiscal expenditure and per capita disposable income, etc. [6] When multiple indicators are adopted, the proportion of each indicator in the constitution of urbanization level is different. Therefore, it is necessary to firstly adopt clustering analysis and analytical hierarchy process (AHP), etc. in statistics to analyse the proportion of each indicator and then obtain the urbanization level after weighting. Since the diversity of the selected indicators, the result obtained with this method is relatively comprehensive and calculation method is rational. Hence, it can comprehensively reflect the urbanization level of certain region or certain city and the conclusion is scientific. However, due to the difference of the research area and research property, selected indicators, methods to obtain data, calculation methods and ratios of various indicators, the urbanization level of the same region may be different and even differ greatly and the vertical comparison of the same region in different years and the horizontal comparison of different regions in the same year are meaningless [10]. Since the retrievable conditions of various indicator data of various regions are different, it is hard to form comparatively general indicator system and measurement method.

Entity is geographic feature and geographic phenomenon in real life and mainly consists of point entity, line entity, surface entity and body entity. Space refers to regular or irregular Euclid space that the length and angle can be measured. Multiple space relationships such as topology, order, distance and orientation exist among entities. What's called entity space is the space structure of entity facilities. Urban entity refers to various urban facilities (for instance, house, building, road and pipeline) and urban landscape mainly consisting of non-farming land and non-farming economic activities. The distribution scope and space structure of these urban facilities and urban landscape are the urban entity space [11]. Here propose urbanization level measurement method of JingJinJi (short for Beijing, Tianjin and Hebei province) Region to calculate the entity space urbanization level and compare with traditional population urbanization level. The research scope is 207 districts and counties inside JingJinJi Region, including 18 districts and counties of Beijing, 18 districts and counties of Tianjin, and 171 districts and counties of 11 prefecture-level cities of Hebei Province [12].

\section{Method and Data}

The measurement method of entity space urbanization rate in JingJinJi Region: "entity space urbanization rate = entity space area/total area of administrative region*100\%". Wherein, the area data of administrative region of districts and counties of JingJinJi Region in 2005 and 2009 were from Chinese County (Urban) Social Economic Statistics Yearbook 2006/2010. The area data of administrative region of Beijing and Tianjin were respectively from Beijing Statistics Yearbook 2006/2010 and Tianjin Statistics Yearbook 2006/2010.

Meanwhile, through using ENVI software to handle MCD12Q1 remote sending image of JingJinJi region in 2000 and 2010, we can obtain vector data of pattern spot of constructed area of JingJinJi region. The handling steps are as below:

(1) Projection transformation: transform data into WGS84 coordinate system.

(2) Transform into vector data: resample the raster data and refer to materials "ENVI analysis on MCD12Q1 data of MODIS product" with resolution rate of $1000 \mathrm{~m}$. According to vegetation classification standard of University of Maryland, if $\mathrm{dn}$ is 13 , it is urban area and constructed area. Extract the remote sensing image data that dn is 13 and it is the area of urban constructed region.

(3) Area calculation of residence zone

After extracting residing area image from MODIS image, transform raster data of residing area into vector data and calculate the area of the residing region. Since the resolution rate of the data is $1000 \mathrm{~m}$, the area of the constructed region should be at least $1 \mathrm{~km}^{2}$. Secondly, the author considers that the area of the constructed region where the urbanized population is distributed is not smaller than $1 \mathrm{~km}^{2}$.

The pattern spot data of 2000 and 2010 JingJinJi Region obtained through processing are as below: 

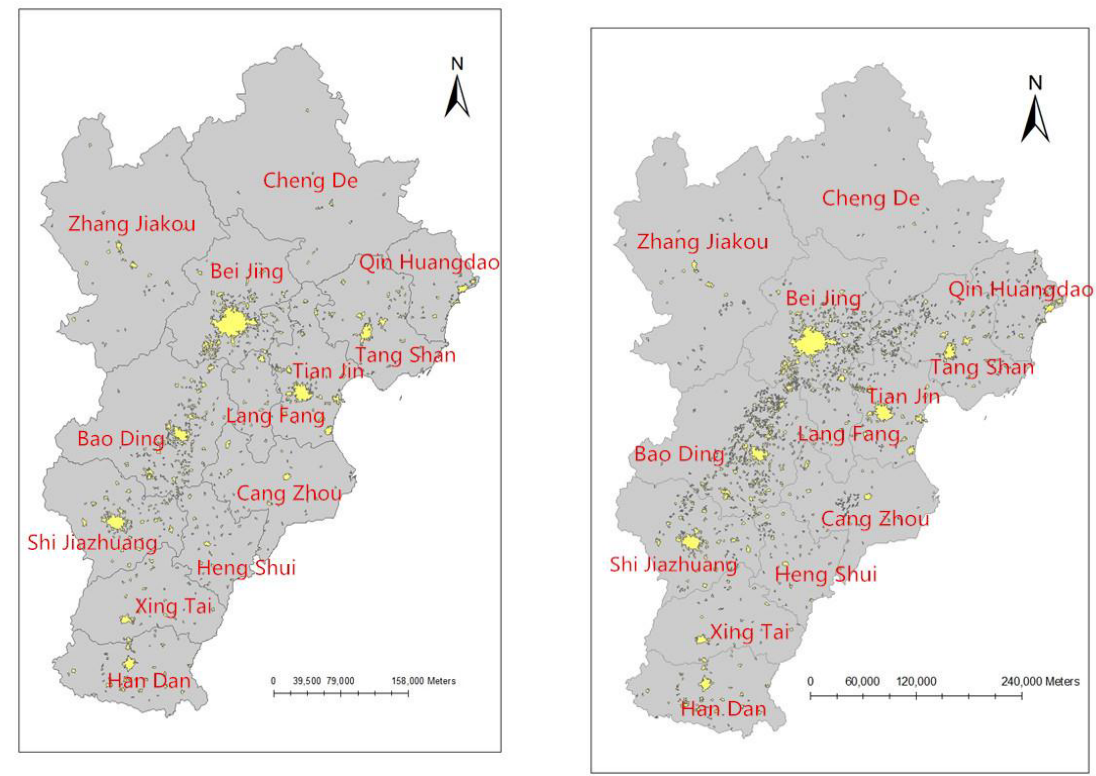

Fig.1. Area of Entity Space of JingJinJi Region in 2005 (Left) and 2009 (Right).

\section{Result}

According to above urbanization rate calculation formula of the entity space, extract the area data of entity space and area data of administrative region of various districts and counties of JingJinJi Region in 2005 and 2009, correct the original data, analyze and process and obtain the urbanization level of the entity space of the districts and counties of JingJinJi Region in 2005 and 2009.
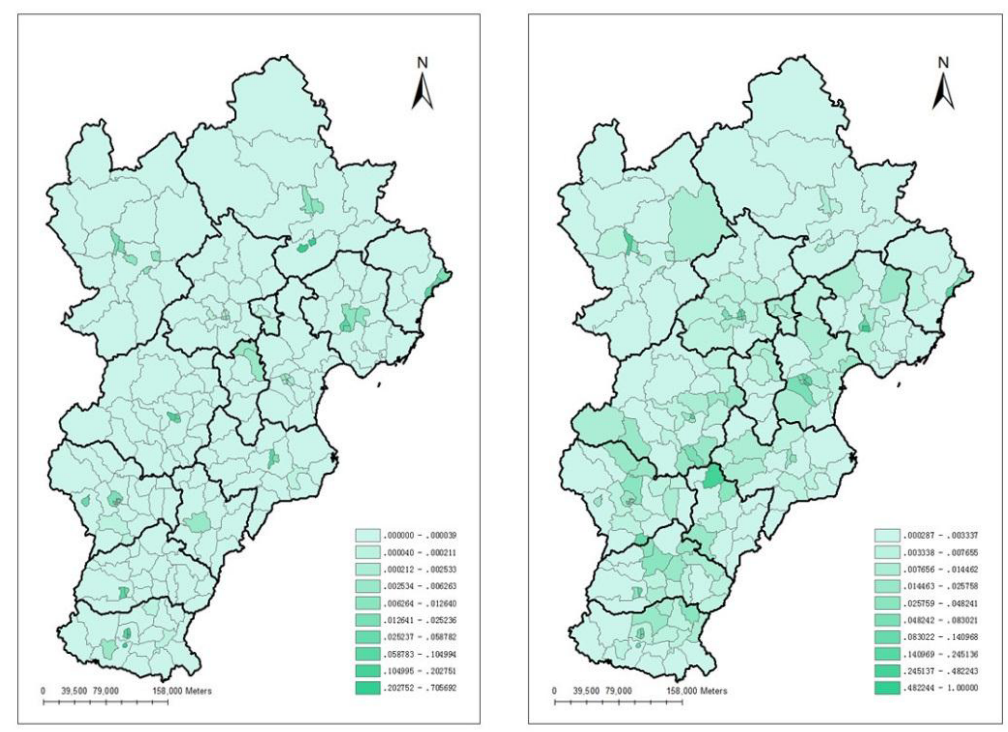

Fig.2. Entity Space Urbanization Rate of Districts and Counties of JingJinJi Region in 2005 (Left) and 2009 (Right).

Among various districts and counties of JingJinJi Region in 2005, the urbanization level of the entity space of the districts and countries was much higher, namely Dongcheng District, Xicheng District, Chongwen District, Xuanwu District (Present Dongcheng District and Xicheng District) of Beijing, Heping District, Hedong District, Hexi District, Nankai District, Hebei District and Hongqiao District of Tianjin, Changan District of Shijiazhuang, Lunan District and Lubei District of Tangshan, Haigang District, Shanhaiguan District and Beidaihe District of Qinghuangdao, Hanshan 
District and Congtai District of Handon, Qiaodong District and Qiaoxi District of Xingtai, Xinshi District, Beishi District and Nanshi District of Baoding, Qiaodong District, Qiaoxi District, Xuanhua District and Xiahuayuan District of Zhangjiakou, Shuangqiao District and Shuangluan District of Chengde, Xinhua District and Yunhe District of Cangzhou, Anci District and Guangyang District of Langfang, and the urbanization level of entity space of other districts and counties was very low.

In 2009, the urbanization level of the entity space of the districts and countries was much higher, namely Dongcheng District, Xicheng District, Chongwen District, Xuanwu District of Beijing, Heping District, Hedong District, Hexi District, Nankai District, Hebei District and Hongqiao District of Tianjin, Changan District, Qiaodong District, Qiaoxi District, Xinhua District and Jingxingkuang District of Shijiazhuang, Lunan District, Lubei District, Guye District and Kaiping District of Tangshan, Haigang District, Shanhaiguan District and Beidaihe District of Qinghuangdao, Hanshan District, Congtai District, Fuxing District and Fengfengkuang District of Handon, Qiaodong District and Qiaoxi District of Xingtai, Xinshi District, Beishi District and Nanshi District of Baoding, Qiaodong District, Qiaoxi District, Xuanhua District and Xiahuayuan District of Zhangjiakou, Shuangqiao District, Shuangluan District, Yingshouyingzikuang District of Chengde, Xinhua District and Yunhe District of Cangzhou, Anci District and Guangyang District of Langfang, Taocheng District of Hengshui and the urbanization level of entity space of other districts and counties was very low.

\section{Discussion}

In the spatial and temporal difference analysis on the urbanization rate of entity space of various districts and countries of JingJinJi, we should firstly analyze according to the time change law, then analyze the space region difference and finally compare the entity space urbanization rate and the population urbanization rate; find out difference in aspects of urbanization order, primacy ratio and zipf indicator and research the distribution features of the entity space urbanization rate.

\subsection{Time change law of entity space urbanization rate}

According to the calculation, the overall entity space urbanization rates of JingJinJi Region in 2005 and 2009 were respectively $0.766 \%$ and $0.782 \%$. It was on the rise but enhanced slowly. The detailed analysis on districts and counties is as below.

(1)The overall change trend of the entity space urbanization rate is stable;

(2)The development of economic and technological development zone has obviously enhanced the entity space urbanization rate;

(3)The change of administrative region is the main reason for the drop of the urbanization rate of some districts and counties.

\subsection{The regional difference of the entity space urbanization rate is obvious}

According to the entity space urbanization level of JingJinJi Region, in consideration of the development features of various districts and counties, and following the high-to-low order of entity space urbanization level, all districts and counties of JingJinJi Region are divided into three teams: central urban area, urban development area and suburb.

Table 1. District Standard of Entity Space Urbanization Rates.

\begin{tabular}{|c|c|}
\hline District Type & $\begin{array}{c}\text { Entity Space Urbanization } \\
\text { Rate Section }\end{array}$ \\
\hline Central Urban Area & {$[10 \%, 1)$} \\
\hline Urban Development Area & {$[1 \%, 10 \%)$} \\
\hline Suburb & $(0,1 \%)$ \\
\hline
\end{tabular}




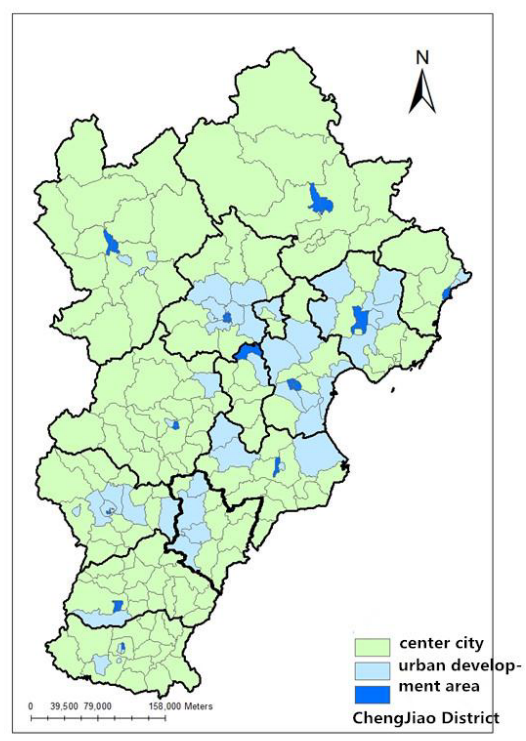

Fig.3. Entity Space Urbanization Rate District Figure of JingJinJi in 2009.

We can found that:

(1)Central urban area has perfect facilities and bears urban core functions.

(2)Urban development area bears urban extension function.

(3)Suburb bears ecological conservation function.

\subsection{Comparison of entity space and population urbanization rate}

During the urbanization course, the population change and space extension are two kinds of expressions [13]. As Chinese urbanization schedule accelerates, the virtual high population urbanization, disordered urban space construction and even out-of-control problem will become gradually extruded [14]. The paradox phenomenon of urban shrinking appears [15]. According to the comparison of entity space urbanization rate and traditional population urbanization rate in the aspects of urbanization order, primacy ratio and zipf indicator, we find the population growth and entity space extension law during the urbanization course in JingJinJi Region.

(1)The population of three teams is inconsistent with the entity space urbanization order.
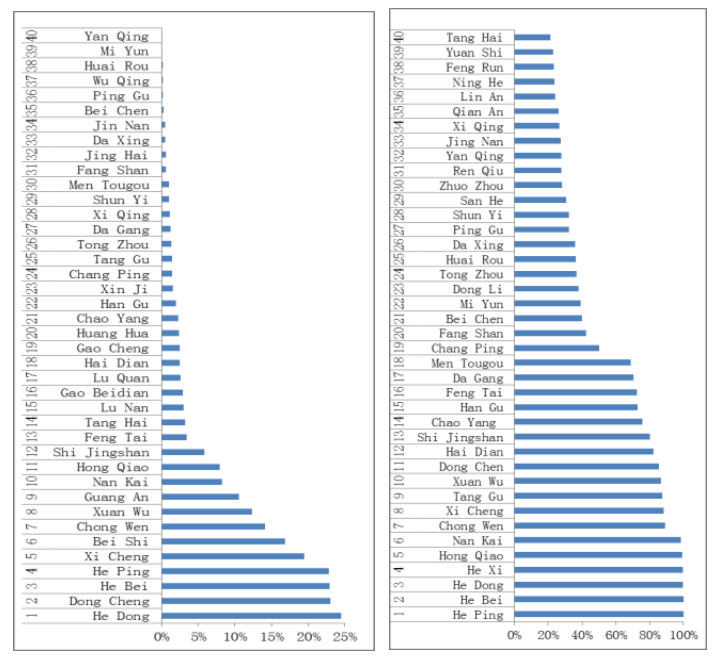

Fig.4. Population (Left) and Entity Space (Right) Urbanization Order (The First 40) of the Districts and Counties of JingJinJi Region in 2009.

(2)The population and entity space urbanization rate of central urban area and suburb is consistent. 


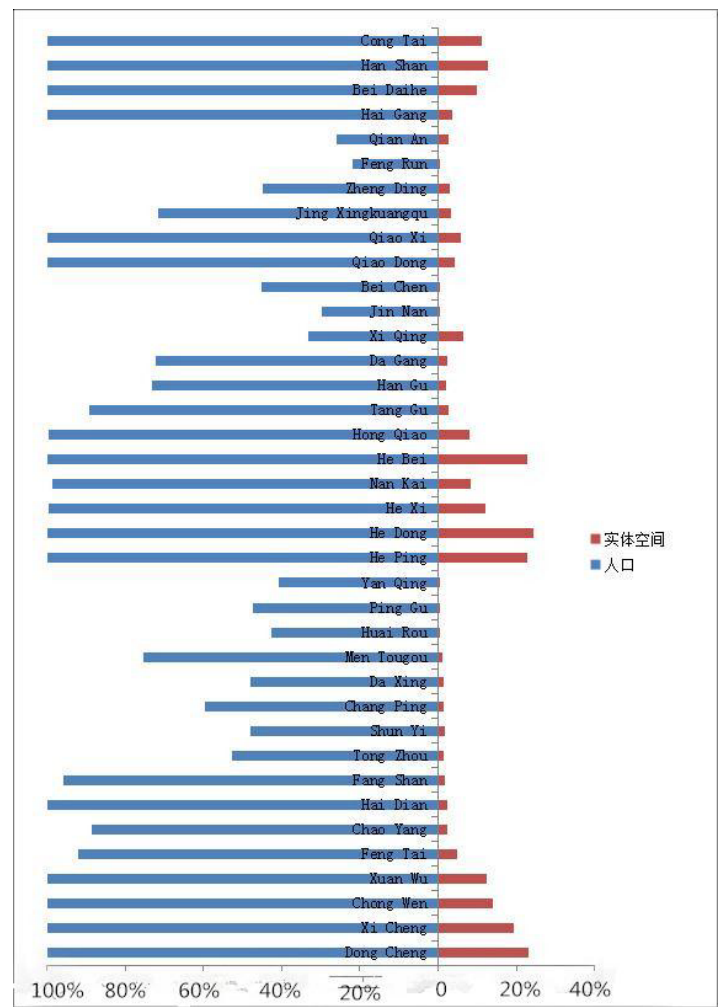

Fig.5. Comparison of Population Urbanization Rate and Entity Space Urbanization Rate of Representative Districts and Counties.

(3)The land use of urban development area is not intensive. The population and entity space urbanization rate in urban development area are not consistent and shows two different types: (1) the entity space urbanization rate is faster than population urbanization rate; (2) the entity space urbanization rate is slower than the population urbanization rate. Now two situations are classified and discussed as below.

(4)The primacy ratio of population urbanization is higher than the primary rate of entity space.

The common index that balances the distribution of the regional city scale is urban primacy ratio and was proposed by American scholar Jefferson at earliest in 1939: "the ratio of the population of the largest city to the population of the second largest city of one country should be the primacy ratio". In the 1980s, the scholars Yuemin Ning and Chongmin Yan introduced the primacy ratio conception into China [16] and later the scholars deepened the conception of the primacy ratio and proposed to reflect the scale of urban other functions in one region with economic primacy ratio, industrial primacy ratio, technological primacy ratio, talent primacy ratio and cultural primacy ratio [17]. Yixing Zhou improved the primacy ratio index and proposed much effective and rigorous four-city index and eleven-city index [18], namely: four-city index(1) and eleven-city index(2),Pn refers to the population scale of the $\mathrm{n}^{\text {th }}$ city.

$$
\begin{aligned}
& S=P 1 /(P 2+P 3+P 4) \\
& S=2 P 1 /(P 2+P 3+\ldots \ldots+P 11)
\end{aligned}
$$

Introduce the conception of the primacy ratio into urbanization level and calculate the primacy ratio of the population and entity space urbanization of JingJinJi Region, listed in the following table. The two-city index of the primacy ratio of the population urbanization of the districts and counties of JingJinJi Region is close to 1, the four-city index is close to $1 / 3$ and the eleven-city index is close to $2 / 11$, meaning that the population urbanization level of JingJinJi Region almost shows "multi-core" status with the second-order districts and counties and also conforming to the fact that the population urbanization rates in the central urban area of Beijing and Tianjin are not very different. However, the two-city index of the primacy ratio of the entity space urbanization is 1.36 and the four-city index and eleven-city index are all higher than the primacy ratio of the population urbanization. The "multi-core" status of the entity space urbanization level is no longer obvious. It reflects that according to the integration degree of the districts and counties, the integration of the population urbanization of the central urban area has tended to maturity, while there is still development space for the integration of the entity space urbanization. 
Table 2. Primacy Ratio of Urbanization of JingJinJi Region.

\begin{tabular}{|l|l|l|}
\hline Index & $\begin{array}{l}\text { Primacy Ratio } \\
\text { of Population } \\
\text { Urbanization }\end{array}$ & $\begin{array}{l}\text { Primacy Ratio of } \\
\text { Entity Space } \\
\text { Urbanization }\end{array}$ \\
\hline $\begin{array}{l}\text { Two-city } \\
\text { Index }\end{array}$ & 1.00032 & 1.36018 \\
\hline $\begin{array}{l}\text { Four-city } \\
\text { Index }\end{array}$ & 0.33392 & 0.47296 \\
\hline $\begin{array}{l}\text { Eleven-city } \\
\text { Index }\end{array}$ & 0.23545 & 0.41486 \\
\hline
\end{tabular}

(5)The zipf index of the entity space urbanization is higher than that of the population urbanization.

Order - the most general scale rule is zipf rule, namely(3). After being changed for logarithm form, it is(4) . Wherein, $\mathrm{Pi}$ is the population of the ith city; $\mathrm{Ri}$ is the city order; q is Zipf index [19]. Through researching the size of the Zipf index, it may reflect the urban scale and form. The larger the q value is, the much uniform the city distribution is. If $\mathrm{q}=+\infty$, the city distribution is equal-size and uniform. The ideal city system according to Zipf should satisfy $\mathrm{q}=1[20]$.

$$
\begin{gathered}
P i=P 1^{*} R i^{-q} \\
L n P i=L n P 1-q L n R i
\end{gathered}
$$

Introduce Zipf rule into urbanization rate and use Pi to express the urbanization rate of the ith city. Adopt formula(5) and regression analysis to respectively calculate the Zipf index q of the population urbanization rate and the entity space urbanization rate in 2005 and work out that the Zipf index of the population urbanization is 0.5051 and the Zipf index of the entity space urbanization is 1.8076 . The zipf index of the entity space urbanization is obviously higher than that of the population urbanization. It means that the scale distribution of the entity space urbanization is much uniform than that of the population urbanization.

$$
L n P i=L n P 1-q L n R i
$$

\section{Conclusion}

On the basis of researching the measurement method of the traditional population, land, economy, society and ecology urbanization level, the paper proposes urbanization level measurement method based on entity space. Calculate the entity space urbanization rates of the districts and counties of JingJinJi Region in 2005 and 2009 according to the formula "urbanization level = area of entity space/area of administrative region*100\%" and analyze the time and spatial difference to work out that the change of the administrative division and the development of the economic and technological development zone are the main factors to influence the change of the entity space urbanization rates of the districts and counties of JingJinJi Region during 2005-2009. Divide the districts and counties of JingJinJi Region into three teams according to the high-to-low order of the entity space urbanization rate: central urban area, urban development area and suburb, and find that the three areas respectively bear different city functions. Compare the entity space urbanization rate and the population urbanization rate of the districts and counties of the three teams in the aspects of order, primacy ratio and zipf, etc. and find the features: in the urban development area of JingJinJi Region, the land use is not intensive; the primacy ratio of the population urbanization is higher than that of the entity space urbanization rate; the entity space urbanization rate scale distribution is much uniform. According to above findings, the paper proposes following advices about the integration development of Beijing-Tianijn-Hebei Region: (1) the space construction should be decided according to the function division. The city division according to the entity space urbanization rate and the function division according to state policy are basically consistent. The central area bears core functions; the development area bears external extension function; the suburb bears ecological conservation function. (2) The urban development area should pay attention to the construction of supporting facilities as well as enhancing the land use rate. Change the extensive land use form of the urban development area, improve infrastructure and meanwhile absorb population through corresponding supporting industries and let population urbanization and entity space urbanization consistent. (3) Enhance city construction and enhance the entity space urbanization rate in small and middle districts and counties surrounding Beijing and Tianjin.

\section{Acknowledgements}

Supported by the Project of The National Natural Science Foundation "Urban Hierarchical System Evolution and Mechanism Study Based on Population Migration Network: Take Beijing-Tianjin-Hebei Region as Example" (No.: 41301178) and the Project of School of National Fiscal Development of Central University of Finance and Economics "Research on Local Governmental Behaviors during Chinese Urbanization Strategy Course” (No: 024050314002/004). 


\section{References}

1. LIU Jiehong,On Urbanization.Journal of Northwest A\&F University(Social Science Edition), 2009,9(4): 58-62.

2. LIU Yaolin,LI Jiwei,HOU Heping, LIU Yan-fang, Study on urbanization rate of urban-rural construction land and its influencing factors: A case study of Hubei Province. Geographical Research,2014,33(1):132-142.

3. GU Chaolin,WU Liya,Productions of the Research of China's Urbanization, Urban Problems,2008(12):2-12.

4. WANG Yang,WANG Shaojian, QIN Jing,Spatial evaluation of land urbanization level and process in Chinese cities,Geographical Research,2014 (12) : 2229-2238.

5. XU Xueqiang, ZHOU Yixing,NING Yue-min, Urban Geography .Bei Jing:Higher Education Press,2010.

6. CHEN Mingxing.Research progress and scientific issues in the field of urbanization. Geographical Research,2015 (4) :614-630.

7. Lu Dadao. The research content framework of urbanization field in Geography. Scientia Geographica Sinica, 2013, 33(8): 897-901.

8. CHEN Mingxing, LU Dadao, ZHANG Hua.Comprehensive Evaluation and the Driving Factors of China's Urbanization. Acta Geographica Sinica,2009(4):387-398.

9. WU Jie,QUAN Shaowei.Discussion of China's population urbanization rate statistics and calculation method. World Research ,2013,(7):44-47.

10. HENG Wen-sheng,WANG Xiao-fang,LI Cheng-gu,the Spatial Disparties of Regional Comprehensive Urbanization level of Vice Province City in China from 1997,Economic Geography, 2007(2).256-260.

11. HU Li. Definition of Urban Proper Scope Based on ETM density of construction land-A Case Study of Dong Guan City.Guang Zhou:South China Normal University.2010.

12. YANG Dongfeng,LONG Ying, YANG Wenshi,SUN Hui,Losing Population with Expanding Space:Paradox of Urban Shrinkage in China. Modern Urban Research.2015(9):20-25.

13. LIU Fawei, XU Hengzhou,WANG Shu,Analysis on Spatial- temporal Coupling Coordinate Degree among Population, Land and Economy Urbanization: Based on China Provincial Panel Data,Urban Development Studies, 2014 (8) : 7-11.

14. YIN Hongling,XU Teng, The Mismatch between Population Urbanization and Land Urbanization in China,Urban Planning Forum, 2013(2): 10-15.

15. LONG Ying, WU Kang,WANG Jianghao. Shrinkage Cities in China, Modern Urban Research, 2015(9):14-19.

16. YAN Zhongmin,NING Yuemin,Changes of the urban population development of China//Research on population, Shang Hai:East China Normal University Press, 1981: 20-37.

17. LU Xuefa,SHEN Huifang. The Status Quo and Countermeasure Research of the first degree of Hang Zhou city Zhejiang Statistics, 2008(6): 32-34.

18. ZHOU Yixing, YANG Qi, A Review on the Urban-size Hierarchy of China and the Territorial Types of the Hierarchy on Provincial Level. Acta Geographica Sinica, 1988, (2): $97-111$.

19. Zipf,G.K. Human Behavior and the principle of Least Effort. Addison-Wesley, Reading, MA.

20. CHEN Kaiming, ZHUANG Yanjie, Spatial Econometric Analysis of The Rank-size Rule for Urban System:A Case of Prefectural-level cities in China's Middle Area, Scientia Geographica Sinica, 2012.8 (8): :905-912. 